\title{
SHORT TIME DYNAMICS OF HIGHLY EXCITED SMALL POLYATOMIC MOLECULES EXTRACTED FROM LASER SPECTRA
}

\author{
KAORU YAMANOUCHI \\ Department of Pure and Applied Sciences, College of Arts and Sciences \\ The University of Tokyo Komaba, Meguro-ku, Tokyo 153, Japan \\ and \\ ROBERT W. FIELD \\ Department of Chemistry, Massachusetts Institute of Technology, Cambridge, \\ Massachusetts 02139, U.S.A.
}

(Received 30 August, 1994)

\begin{abstract}
The recent spectroscopic investigations of vibrational dynamics of highly excited acetylene $\left(\mathrm{C}_{2} \mathrm{H}_{2}\right)$, predissociation of vibronically excited $\mathrm{NO}_{2}$, and direct dissociation of OCS in the deep vacuum ultraviolet region are introduced. It is demonstrated that (i) rich and specific information on the excited state dynamics is encoded in various types of observed spectra such as stimulated emission pumping, dispersed fluorescence, laser induced fluorescence, absorption, and photofragment excitation spectra and that (ii) the early stage dynamics after photoexcitation can be extracted from observed spectra by the recently developed techniques, i.e. feature state assignments, a superpolyad Hamiltonian model, the extended autocorrelation method, and hierarchical, statistical and Fourier transform analysis methods.
\end{abstract}

KEY WORDS: Feature state assignments, Short time dynamics, IVR, Unimolecular reactions, Photodissociation, PHOFEX spectroscopy

\section{INTRODUCTION}

In this decade, advances in laser spectroscopic techniques have revealed the level structure of polyatomic molecules at chemically significant energies near or above the threshold for unimolecular isomerization and dissociation reactions [1-3]. An early study of the stimulated emission pumping (SEP) spectrum of acetylene $\left(\mathrm{C}_{2} \mathrm{H}_{2}\right)$ by Field and coworkers [4] greatly stimulated both experimental and theoretical investigations of vibrationally highly excited small polyatomic molecules. In such highly excited energy regions, molecular spectra exhibit extremely dense transition peaks and/or complicated peak distributions, as was shown for acetylene and $\mathrm{SO}_{2}$ [5]. A conventional spectroscopic analysis, such as an assignment of quantum num- 
bers to each of the observed peaks, seemed to be impossible. Therefore, some statistical analysis methods, which had been developed long ago by nuclear physicists [6], were applied and the statistical properties derived from an observed complicated molecular spectrum were proposed as characteristic of chaotic vibrational dynamics. This proposal was based on the similarity of the observed spectrum to the statistical characteristics that can be derived from the level structure of a quantum non-linearly coupled oscillator in the energy region where the corresponding classical oscillator exhibits chaos. It was often discussed how close the spectrum approaches to the chaotic limit in terms of statistical measures such as the Brody parameter for the nearest neighbor level spacing distribution, $\Sigma^{2}$ and $\Delta_{3}$ statistics, and the number of degrees of freedom from a $x^{2}$ intensity distribution $[6,7]$. However, there was frustration among molecular spectroscopists when they heard about "chaotic" spectra because no molecule-specific information can be derived from such statistical properties. The situation in molecular spectroscopy was different from that in nuclear physics. Since we know that molecular vibrations are well described by solving Schrödinger's equation for a ball and spring model, it is more substantial and exciting to understand well-defined mechanisms such as anharmonic resonances, which mix these vibrational motions and may eventually produce such chaotic character.

It is clear that vibrational assignments can be performed without ambiguity for vibrational levels in the low energy region, where vibrational levels are regularly spaced in a manner described by a Dunham type expansion. Therefore, if it were possible to extend secure vibrational assignments to higher energy, the specific mechanisms that destroy the regularity of spectral patterns could be identified. Based on this naive idea, a dispersed fluorescence (DF) spectrum was recorded over a wide energy region of the electronic ground state of $\mathrm{SO}_{2}$ and vibrational assignments were extended toward the highly excited region [8]. In the case of $\mathrm{SO}_{2}$, transition peaks in the DF spectrum representing vibrational levels could be assigned, even in the vibrationally highly excited region, by using a third-order Dunham expansion. However, comparison of the DF spectrum, whose spectral resolution is relatively low $\left(\sim 4 \mathrm{~cm}^{-1}\right)$, and the SEP spectrum measured with high resolution $\left(\sim 0.5 \mathrm{~cm}^{-1}\right)$ showed that each peak assigned by a set of vibrational quantum numbers in the DF spectrum actually splits into several peaks at the higher resolution of an SEP spectrum. These finer peaks could not be vibrationally assigned. This observation, that a set of quantum numbers can be assigned to an unresolved cluster of eigenstates, was later developed into the feature state assignment scheme in the course of the analysis of the DF spectra of acetylene.

In the present article, we introduce several recent advances in the spectral analysis of polyatomic molecules at chemically significant vibrational energies by referring to the studies of (i) the vibrational dynamics and the isomerization reaction of highly excited acetylene, (ii) IVR and predissociation dynamics of $\mathrm{NO}_{2}$ and (iii) direct dissociation of OCS on a repulsive potential energy surface (PES), and we describe how the idea of feature state assignments and other techniques, which were developed in the course of the analysis of SEP and DF spectra of acetylene, afforded a basis to extract dynamical behavior from the observed spectra of small polyatomic molecules for vibrational excitation energies both above and below the reaction 
threshold. Throughout these studies, an emphasis will be placed on how rich and substantial information on the short time dynamics is encoded in the observed spectra of polyatomic molecules.

We describe first in Section II how the complex acetylene spectrum in its vibrationally excited region was clarified by the combined analysis of the SEP and DF spectra [9]. It will be shown that the idea of "feature state assignments" was found to be well-suited to describe a stepwise intramolecular vibrational energy redistribution (IVR) of the highly excited acetylene and to explicitly describe the energy flow pathway from the assigned Franck-Condon (FC) bright state toward eigenstates. $[9,10]$. A series of new techniques and new ideas were developed to handle the level structure of highly excited acetylene. In addition to the feature state assignment scheme, a statistical-Fourier transform analysis $[11,12]$, a spectral cross-correlation analysis [13], a tree (or hierarchical) analysis [14-16], an extended autocorrelation (XAC) analysis [16], and a superpolyad Hamiltonian approach [3] were all applied to the SEP and/or DF spectra of acetylene. Some of these analyses will also be introduced in Section II.

In Section III, we introduce the vibronic level structure of $\mathrm{NO}_{2}$ below its first dissociation limit, as observed by laser induced fluorescence (LIF) spectroscopy [17], and its photodissociation mechanism, as investigated by photofragment excitation (PHOFEX) spectroscopy [18]. We will describe a mechanistic picture of the short time dynamics after the excitation in the ultraviolet (UV) wavelength region that can be extracted by assignments of feature states, which were identified by a convolution analysis of the LIF spectrum. It will be shown that the level statistics, measured at energies just below the dissociation threshold, and the RRKM behavior of the predissociation reaction observed at energies just above the threshold, afforded a rare opportunity to relate the chaotic level distribution to the assumption of chaos behind the statistical unimolecular reaction rate theory.

In Section IV, by introducing recent measurements of the PHOFEX spectrum of OCS in the deep VUV region [19], we will show that information about the short time dynamics on the repulsive PES can be deduced from intrinsically broadened feature states via the unstable periodic orbits which are inferred from the Fourier transform of absorption and/or PHOFEX spectra.

\section{FEATURE STATE ASSIGNMENTS AND SPECIFIC IVR MECHANISM OF ACETYLENE}

By measuring SEP spectra of acetylene in the $26500 \mathrm{~cm}^{-1}$ region at two different spectral resolutions, Pique et al. identified two time scales of the vibrational dynamics based on the statistical Fourier transform of the SEP spectra [11a], and the difficulty in extracting the vibrational dynamics by statistical analyses was discussed later [11b, c]. The recurrences found in the Fourier transformed profile were suggested to be a signature of the large amplitude vibrational motion of one $\mathrm{H}$ atom around the $\mathrm{CCH}$ core [12]. At that stage, it was not clear whether the large amplitude motion really exists nor could the mechanism which promotes such a vibration be specified from 
the spectral analysis. However, being stimulated by these SEP studies by Field and co-workers at MIT and guided by the naive idea obtained from the SEP and DF spectra of $\mathrm{SO}_{2}$, the DF spectra of acetylene over a wide energy region were measured by Yamanouchi, Tsuchiya and coworkers at University of Tokyo (Komaba Campus) and both SEP and DF spectra of vibrationally highly excited acetylene were jointly analyzed in a trans-Pacific collaboration [21]. It was this collaborative study which afforded an opportunity to identify and specify the stepwise IVR mechanism in vibrationally highly excited acetylene.

Yamanouchi et al. [9] performed a vibrational assignment of the DF spectrum and proposed feature state assignments for a group of levels in the vibrationally highly excited region. It was found that there are hierarchical level structures in the region above $14000 \mathrm{~cm}^{-1}$ and two stages of hierarchy were found in the DF spectrum and a third stage of hierarchy was found by the comparison of the DF and SEP spectra. The hierarchical level structure was interpreted as stepwise IVR and a model based on the feature state assignments of the DF peaks was able to specify the energy flow pathway in the IVR process. In the feature state assignment scheme, a set of quantum numbers is assigned to a feature state, a group of eigenstates, which represents a shorter time dynamics, and the observed hierarchical level structure was interpreted in terms of stepwise energy flow. Based on the feature state assignments of the hierarchical structure, it was proposed that the energy flows in a stepwise manner from an initially excited Franck-Condon (FC) bright state, of dominant $v_{2}$ (CC stretch) and $v_{4}$ (trans bend) character, to an originally dark $v_{5}$ (cis bend) mode by DarlingDennison and vibrational $\ell$-resonances, and then to the $v_{3}$ (antisymmetric $\mathrm{CH}$ stretch) mode by a 2345 anharmonic resonance. The idea of the tier of Darling-Dennison resonances proposed in the interpretation of the feature states in the DF spectra later became known as a super-polyad. In other words, it was demonstrated first that the intramolecular vibrational energy redistribution pathway can be extracted and specified by the assignments of feature states in a complex molecular spectrum $[9$, 10]. The IVR process identified in the DF spectrum was later confirmed by the higher-resolution SEP spectrum in the lower energy region around $7000 \mathrm{~cm}^{-1}$ [10].

The detailed spectroscopic analysis of the SEP spectrum led to the idea of a superpolyad Hamiltonian [3]. A traditional multi-resonance superpolyad effective Hamiltonian matrix was constructed to describe the coarse structure in the spectrum and the early time intramolecular dynamics. Since this superpolyad model is based on matrix elements of a relatively small number of anharmonic coupling terms evaluated in a normal mode, harmonic oscillator, product basis set, it can be readily scaled to higher energy and provide an accurate model for early time sequential IVR processes. Upon scaling to higher energy, the model provides testable predictions as the early time dynamics become more rapid and more complex.

On the other hand, being stimulated by the successful interpretation of the hierarchical structure in the SEP and DF spectra in terms of the feature state assignments, a tree-based hierarchical analysis was proposed as a method in which a hierarchy of coupling matrix elements can be deduced [14-16]. The tree analysis $[14,15]$ and the parsimonious tree analysis [16] were applied first to the LIF spectrum of $\mathrm{NO}_{2}$ in the UV wavelength region. Later, another type of hierarchical analysis called a 
convolution analysis was applied to the DF spectrum of acetylene [2]. It is true that the level hierarchy could be related to some specific dynamics encoded in the spectrum, but the mechanism of the dynamics specific to the molecule under study could not be identified only from such a hierarchical analysis.

The extended autocorrelation (XAC) analysis [16] is also a new procedure aiming to extract automatically a certain pattern such as doublets or triplets without relying on the skill and patience of spectroscopists. Using the DF spectrum of acetylene, it was demonstrated that the XAC analysis could recognize a characteristic pattern which had been previously assigned as feature states.

Recently various techniques of spectral analysis were categorized into three types [2]; (i) a feature state assignment as a generalized conventional spectroscopic assignment, (ii) statistical analysis, and (iii) hierarchical analysis, and their characteristics and applicabilities were discussed and a systematic and efficient procedure was proposed to extract dynamics from an observed spectrum such as DF and SEP spectra. According to this procedure, the first step is to find good quantum numbers that describe the shortest time dynamics. Then, by improving the resolving power gradually, good quantum numbers for the next lower level of the hierarchy are sought, and consequently the stepwise energy flow pathway from the FC bright states is specified. When we reach the stage in the hierarchy of finer and finer feature states at which the definite assignment becomes impossible, statistical analyses are performed to quantitate the extent of the IVR.

As mentioned above, the Fourier transformed profile of the SEP spectrum of acetylene showed a characteristic recurrence structure. This recurrence was interpreted as a large amplitude vibrational motion of one $\mathrm{H}$ atom around the $\mathrm{CCH}$ core [12], which is the high energy residue of the isomerization reaction from acetylene ( $\mathrm{HCCH})$ to vinylidene $\left(\mathrm{CCH}_{2}\right)$. This large amplitude motion can be related to the strong Darling-Dennison resonance between the two degenerate bending modes, as identified by the feature state assignments and by the deperturbation analysis in the lower energy region. The DD and the vibrational $\ell$-resonance mechanism promotes substantial mixing of two bending modes, which eventually results in a local bending motion of the $\mathrm{H}$ atom with respect to the $\mathrm{CCH}$ core in the highly excited region. In later theoretical work, the specific local bending states were identified, whose vibrational wavefunction is directed toward the vinylidene configuration [21]. It is interesting to note that the existence of a classical orbital motion of the $\mathrm{H}$ atom in the highly excited region of acetylene was suggested independently by different experimental approaches as well as by several theoretical studies. This classical motion may be regarded as a classical periodic orbit [22] and scars of classical periodic orbits [23] embedded in the complex spectrum in the classically chaotic regime.

\section{GOE STATISTICS OF $\mathrm{NO}_{2}$ LEVEL STRUCTURE AND RRKM THEORY}

It is well-known that $\mathrm{NO}_{2}$ exhibits an extremely complex LIF spectrum in the visibleUV regions. Despite enormous effort, very little useful information has been extracted based on conventional spectral assignments. In order to analyze the complex vibronic 
spectrum of $\mathrm{NO}_{2}$, feature state assignments were performed over a wide energy region below the dissociation threshold [17]. The well-resolved high resolution spectrum was found to be very complicated due to vibronic and rovibronic couplings between the electronic ground ${ }^{2} \mathrm{~A}_{1}$ state and the FC bright ${ }^{2} \mathrm{~B}_{2}$ state, and it seemed impossible to assign a set of vibrational quantum numbers to each of these eigenstates. However, by convoluting the high resolution spectrum, it was found that zero-order FC bright features, which represent the bending progression of the ${ }^{2} \mathrm{~B}_{2}$ state, could be identified. Based on this feature state assignment, it was shown that IVR occurs first from an initially bright bending mode of the ${ }^{2} \mathrm{~B}_{2}$ state to the remaining two vibrational modes within the ${ }^{2} \mathrm{~B}_{2}$ manifold, and finally, the vibronic interaction promotes energy flow toward the three vibrational modes in the vibrationally highly excited region of the ground ${ }^{2} \mathrm{~A}_{1}$ state. However, it was impossible to identify the specific resonances which mix the vibrational modes in both the ${ }^{2} \mathrm{~B}_{2}$ and ${ }^{2} \mathrm{~A}_{1}$ states.

Instead of assigning a less pronounced hierarchical structure, three types of statistical analyses were performed in the region just below the dissociation threshold. The nearest neighbor level spacing distribution, which represents the short-range correlation of an eigenstate spectrum, showed that the correlation is extremely strong and its magnitude is close to the limit of an eigenstate spectrum of Hamiltonian matrices forming a Gaussian orthogonal ensemble (GOE). Furthermore, the results from intensity distribution and Fourier transform analyses consistently showed that the level structure of $\mathrm{NO}_{2}$ in the energy region just below the dissociation threshold exhibits a GOE type correlation. Indeed, in the Fourier transformed spectra, a large correlation hole, whose recovery time is almost equal to the reciprocal of the density of states, was found. In this case no significant recurrence was evident probably due to the fact that anharmonic couplings and the vibronic interactions mix the zero-order states almost completely. These results of statistical analyses indicate that an almost complete IVR occurs in the energy region just below the dissociation threshold, and that a similar extent of IVR is expected in the energy region just above the dissociation threshold, where no fluorescence can be detected due to the rapid unimolecular reaction. The complete IVR has significance when one thinks of unimolecular dissociation. In the statistical theory of unimolecular dissociations, i.e. RRKM theory, a complete IVR prior to dissociation is prerequisite to the RRKM expression for the unimolecular dissociation rate. Therefore, the statistical analysis of the level structure just below the dissociation limit suggests that the dissociation reaction occurs statistically and its rate can be predicted by the RRKM theory.

The vibronic level structure just below the dissociation threshold was further utilized for the interpretation of the unimolecular reaction dynamics of $\mathrm{NO}_{2}$ just above the dissociation threshold [17]. The photodissociation reaction of $\mathrm{NO}_{2}$ just above the dissociation threshold was investigated by photofragment excitation (PHOFEX) spectroscopy [18], where the wavelength of the laser used for the photodissociation is scanned while the population of a preselected photofragment level is monitored by LIF by another wavelength-tuned laser [24]. The PHOFEX spectrum obtained for $\mathrm{NO}_{2}$ showed that the reaction rate increases as a function of the excitation energy in a stepwise manner as successive energetically allowed channels open. This observation was regarded as a direct confirmation that the photodissociation reaction 
proceeds as predicted by the RRKM theory, which predicts the unimolecular reaction rate to vary as $\mathrm{k}=\mathrm{W} /(\mathrm{h} \rho)$, where $\mathrm{W}$ represents a number of energetically open channels at the transition state, and $\rho$ is the vibrational density of states of the parent molecule. In this case, $\mathrm{W}$ was counted as the number of energetically allowed states of the two fragments, $\mathrm{NO}$ and $\mathrm{O}$, by locating the transition state at infinite separation. Fluctuations, observed in the branching ratios for quantum states of NO fragments, were regarded as additional evidence indicating that the wavefunctions of the quasibound states are statistical mixtures of the zero-order states.

The word "chaos" has attracted many spectroscopists and shed light on the importance of quantum non-linear dynamics in understanding polyatomic molecules. However, sometimes the word chaos provoked misunderstanding that the dynamics could be described satisfactorily by statistical measures. However, in the case of vibronic dynamics of $\mathrm{NO}_{2}$, the evidence of chaotic level structure has significance in the sense that it forms a bridge between the level structure in the highly excited region and the theory of statistical unimolecular reactions.

\section{PHOFEX SPECTROSCOPY OF OCS AND UNSTABLE PERIODIC ORBITS}

When polyatomic molecules absorb a photon in the vacuum ultraviolet (VUV) $(<200$ $\mathrm{nm}$ ) wavelength region, a photodissociation reaction occurs in most cases, because the lowest dissociation threshold is usually located at much lower energy. The photodissociation of simple polyatomic molecules in the VUV region is important to understand photochemistry in the troposphere and stratosphere, because the radicals produced after photodissociation initiate chain reactions, which govern the concentrations of atomic molecular species in the atmosphere [25]. It is interesting to note that some absorption bands of simple polyatomic molecules in the VUV region exhibit a relatively sharp progression despite rapid dissociation processes which usually broaden absorption peaks [26]. For example, in the 180-160 nm region, the absorption spectrum of OCS shows extensive vibrational structure, which has been ascribed to a progression in mainly the bending vibration of the excited ${ }^{1} \Pi$ state in a ${ }^{1} \Pi-{ }^{1} \Sigma$ electronic transition. The ${ }^{1} \Sigma-1 \Sigma$ absorption spectrum of OCS, located at higher photon energies $(160-140 \mathrm{~nm})$, is simpler and exhibits vibrational progressions in the symmetric and antisymmetric stretching vibrations of the excited $1 \Sigma$ state $[27,28]$.

As demonstrated in the analysis of the absorption features of ozone by Johnson and Kinsey [29], the Fourier transform of an absorption spectrum can be related to unstable periodic orbits on the repulsive PES. Similar analyses were performed for the absorption spectrum of $\mathrm{H}_{2} \mathrm{O}[30,31]$ and $\mathrm{H}_{2} \mathrm{~S}$ [31] and for the SEP spectra of $\mathrm{Na}_{3}$ and $\mathrm{HCN}$ [32], and unstable periodic orbits were also discussed theoretically for $\mathrm{CO}_{2}$ [33] and $\mathrm{HgI}_{2}$ [34]. Considering that the progression of the absorption features in a ${ }^{1} \Sigma-1 \Sigma$ transition of OCS exhibits a well separated sharp structure as compared with the absorption spectra for $\mathrm{O}_{3}, \mathrm{H}_{2} \mathrm{O}$, and $\mathrm{H}_{2} \mathrm{~S}$, it is likely that some clear and distinct recurrences will appear in the autocorrelation function obtained by 
the Fourier transformation of the absorption spectrum. Recently, Ohde et al. [35] derived the autocorrelation function for the jet-cooled absorption features recorded by McCarthy and Vaida [28] as shown in Fig. 1. The autocorrelation function revealed the existence of two unstable periodic orbits which start near the transition state region of the PES. One unstable periodic orbit, which has the shorter period of $42 \mathrm{fs}$, was regarded as a symmetric stretching motion and the other, with a $84 \mathrm{fs}$ period, may represent a motion which has a substantial contribution from an antisymmetric vibrational motion. It is interesting to note that the dissociation dynamics of $\mathrm{CO}_{2}$, which is isoelectronic with OCS, on the corresponding PES has a shortest period unstable periodic orbit of $38 \mathrm{fs}$ [33], which is comparable with the $42 \mathrm{fs}$ period of OCS.

On the other hand, by measuring the PHOFEX spectrum of OCS, Pibel, Ohde and Yamanouchi [19] demonstrated recently the capability of using coherent VUV light [36], the so-called VUV laser, generated by the two-photon resonant four wave mixing technique, as a tunable photolysis light source. The broad tunability, high resolution, and high intensity of the VUV source were combined with the cold conditions of a free jet expansion to measure the PHOFEX as well as Doppler spectra of the $\mathrm{S}\left({ }^{1} \mathrm{~S}\right)$ fragment atom. The PHOFEX spectrum near $154 \mathrm{~nm}$ was better resolved than previous absorption spectra and an upper limit for the dissociation lifetime was derived to be 0.26 ps. Therefore, it can be said that OCS oscillates several times along the shortest period unstable periodic orbit within the dissociation lifetime. In other words, the wavepacket as a whole evolves into the product valley as time elapses while the remnant of the wavepacket oscillates along the symmetric stretching coordinate starting from the FC point, as discussed by Schinke [33]. By assigning unstable periodic orbits, the shape of the PES near the transition state region could

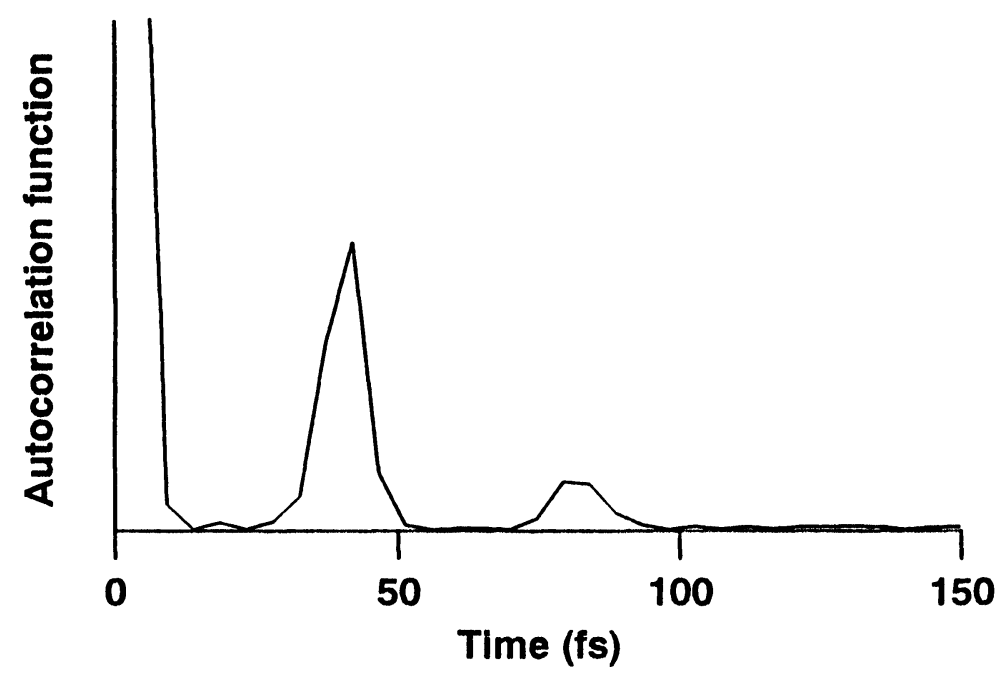

Figure 1 An autocorrelation function obtained from Fourier transformation of the ${ }^{\prime} \Sigma-{ }^{\prime} \Sigma$ absorption band of OCS in the 142.7-158.9 nm region. The jet-cooled absorption spectrum recorded by McCarthy and Vaida (Ref. 28) was used. 
be deduced. It was demonstrated that a structured absorption spectrum in the VUV region carries information about the early stage dynamics of the photodissociation and such dynamics can be characterized by the classical unstable periodic orbits extracted from the absorption feature. It should be noted that the Fourier transform can specify the dynamics on the dissociative PES only when the recurrences are related to the period of a certain unstable periodic orbit, or synonymously, the absorption spectral features are assigned in terms of some vibrational motion.

\section{SUMMARY AND CONCLUSION}

Recent spectroscopic studies, aiming to extract the early time dynamics of acetylene, $\mathrm{NO}_{2}$, and OCS, were introduced. Referring to spectroscopic studies of acetylene, it was shown how the excited state dynamics of polyatomic molecules can be extracted from observed spectra which contain rich dynamical information about processes such as intramolecular vibrational energy redistribution (IVR), isomerization, and photodissociation.

The shorter time dynamics is encoded in the lower resolution spectrum, and broader feature states can specify an earlier stage of the energy flow. This relationship between the frequency and time domains is described by a Fourier transformation. Based on the Fourier transformation of the observed spectrum, large amplitude motions in highly excited acetylene, complete IVR of $\mathrm{NO}_{2}$, and unstable periodic orbits of OCS were characterized. In the case of vibrationally highly excited acetylene, the existence of a large amplitude motion of one $\mathrm{H}$ atom around the $\mathrm{CCH}$ core, which is related to acetylene $\leftrightarrow$ vinylidene isomerization, was suggested by the feature state assignments of the DF spectrum, by the detailed spectroscopic analysis, and by the Fourier transform of the SEP spectrum.

The hierarchical and statistical analyses of the vibronic level structure of $\mathrm{NO}_{2}$ showed that IVR proceeds from the initially excited FC bright bending mode to other modes and, successively, the vibronic mixing occurs to form an eigenstate spectrum that reflects complete IVR. PHOFEX spectroscopy of $\mathrm{NO}_{2}$ just above the dissociation threshold exhibited a dissociation rate which obeys RRKM theory. This behavior of the unimolecular reaction was consistent with complete IVR, as identified in the energy region just below the dissociation threshold. The existence of unstable periodic orbits, which characterize an early stage of the dynamics on the repulsive potential energy surface, can be extracted by the Fourier transform of the PHOFEX spectrum of dissociating OCS in the deep VUV region.

\section{Acknowledgements}

K. Y. is grateful to Professor Soji Tsuchiya, Emeritus Professor of University of Tokyo for the helpful and valuable discussion which stimulated and guided the author's interest toward the dynamics of highly excited polyatomic molecules. The author also thanks Dr. Akiyoshi Hishikawa and Ms. Kyoko Ohde for their assistance in the Fourier transform analysis. The authors thank Professor Koji Kaya for provid- 
ing them an opportunity to contribute to this special issue of Laser Chemistry honoring Professor S. Tsuchiya.

\section{References}

1. (a) K. Yamanouchi, in "Molecular Dynamics and Spectroscopy by Stimulated Emission Pumping", edited by H. L. Dai and R. W. Field (World Scientific, Singapore, 1994) (in press); (b) K. Yamanouchi and S. Tsuchiya, in “Dynamics of Excited Molecules", edited by K. Kuchitsu, p. 217 (Elsevier, Netherlands, 1994).

2. K. Yamanouchi, J. Miyawaki, S. Tsuchiya, D. M. Jonas, and R. W. Field, Laser Chemistry 14, 183 (1994).

3. R. W. Field, S. L. Coy, and S. A. B. Solina, Prog. Theoret. Phys., Suppl. 116, 143 (1994).

4. (a) E. Abramson, R. W. Field, D. Imre, K. K. Innes, and J. L. Kinsey, J. Chem. Phys. 80, 2298 (1984); (b) J. Chem. Phys. 83, 453 (1985).

5. (a) K. Yamanouchi, H. Yamada, and S. Tsuchiya, J. Chem. Phys. 88, 4664 (1988); (b) K. Yamanouchi, S. Takeuchi, and S. Tsuchiya, Prog. Theoret. Phys. Suppl. 98, 420 (1989).

6. (a) T. A. Brody, J. Flores, J. B. French, P. A. Mello, A. Pandey, and S. S. M. Wong, Rev. Mod. Phys. 53, 385 (1981); (b) "Statistical Theories of Spectra: Fluctuations", edited by C. E. Porter, (Academic, New York, 1965); (c) M. L. Metha, "Random Matrices and the Statistical Theory of Energy Levels", (Academic, New York, 1967).

7. (a) T. Terasaka and T. Matsushita, Phy. Rev. A 32, 538 (1985); (b) D. W. Noid, M. L. Koszykowski, and R. A. Marcus, Ann. Rev. Phys. Chem. 32, 267 (1981); (c) Th. Zimmermann, L. S. Cederbaum, H.-D. Meyer, and H. Köppel, J. Phys Chem. 91, 4446 (1987).

8. K. Yamanouchi, S. Takeuchi, and S. Tsuchiya, J. Chem. Phys. 92, 4044 (1990).

9. K. Yamanouchi, N. Ikeda, S. Tsuchiya, D. M. Jonas, J. K. Lundberg, G. W. Adamson, and R. W. Field, J. Chem. Phys. 95, 6330-6342 (1991).

10. (a) D. M. Jonas, S. A. B. Solina, B. Rajaram, R. J. Silbey, R. W. Field, K. Yamanouchi, and S. Tsuchiya, J. Chem. Phys. 97, 2813-2816 (1992), (b) J. Chem. Phys. 99, 7350-7370 (1993).

11. (a) J.-P. Pique, Y. Chen. R. W. Field, and J. L. Kinsey, Phys. Rev. Lett. 58, 475 (1987); (b) J. Wilkie and P. Brumer, Phys. Rev. Lett. 69, 2018 (1992); (c) J.-P. Pique, Y. Chen, R. W. Field, and J. L. Kinsey, Phys. Rev. Lett. 69, 2019 (1992).

12. J.-P. Pique, M. Lombardi, Y. Chen, R. W. Field, and J. L. Kinsey, Ber. Bunsenges. Phys. Chem. 92, 422 (1988).

13. Y. Chen, D. M. Jonas, C. E. Hamilton, P. G. Green, J. L. Kinsey, and R. W. Field, Ber. Bunsenges. Phys. Chem. 92, 329 (1988); (b) Y. Chen, D. M. Jonas, J. L. Kinsey, and R. W. Field, J. Chem. Phys. 91, 3976 (1989).

14. (a) M. Davis, Chem. Phys. Lett. 192, 479 (1992); J. Chem. Phys. 98, 2614 (1993).

15. M. Davis in "Molecular Dynamics and Spectroscopy by Stimulated Emission Pumping", edited by H. L. Dai and R. W. Field (World Scientific, Singapore, 1994) (in press).

16. S. Coy, D. Chasman, and R. W. Field, in "Molecular Dynamics and Spectroscopy by Stimulated Emission Pumping", ditated by H. L. Dan and R. W. Field (World Scientific, Singapore, 1994) (in press).

17. J. Miyawaki, K. Yamanouchi, and S. Tsuchiya, J. Chem. Phys. 101 (1994) in press.

18. J. Miyawaki, K. Yamanouchi, and S. Tsuchiya, J. Chem. Phys. 99, 254 (1993).

19. C. D. Pibel, K. Ohde, and K. Yamanouchi, J. Chem. Phys. 101, 836 (1994).

20. R. W. Field, Laser Chemistry (this special volume) (1994).

21. E. L. Sibert, and R. C. Mayrhofer, J. Chem. Phys. 99, 937 (1993).

22. M. C. Gutzwiller, Chaos in Classical and Quantum Mechanics (Springer-Verlag, New York, 1990).

23. E. J. Heller, in "Chaos and Quantum Physics, NATO Les Houches Lecture Notes", edited by A. Voros, M. Gianonni, and O. Bohigas, p. 547 (Noth-Holland, Amsterdam, 1990).

24. W. H. Green, C. B. Moore, and W. F. Polik, Ann. Rev. Phys. Chem. 43, 591 (1992).

25. R. P. Wayne, Chemistry of Atmospheres, 2nd Ed. p. 27 (Oxford University Press, Oxford, 1991).

26. H. Okabe, Photochemistry of Small Molecules, (John Wiley and Sons, New York, 1978).

27. J. W. Rabelais, J. M. McDonald, V. Scherr, and S. P. McGlynn, Chem. Rev. 71, 73 (1971).

28. M. I. McCarthy and V. Vaida, J. Phys. Chem. 92, 5875 (1988).

29. (a) B. R. Johnson and J. L. Kinsey, Phys. Rev. Lett. 62, 1607 (1989); J. Chem. Phys. 91, 7638 (1989). 
30. N. E. Henriksen, J. Zhang, and D. G. Imre, J. Chem. Phys. 89, 5607 (1988).

31. L. J. Butler, Chem. Phys. Lett. 182, 393 (1991).

32. J. M. Gomez-Llorente, S. C. Farantos, O. Hahn, and H. S. Taylor, J. Opt. Soc. Am. B, 7, 1851 (1990).

33. (a) R. Schinke and V. Engel, J. Chem. Phys. 93, 3252 (1990); (b) R. Schinke, Photodissociation Dynamics, (Cambridge University Press, Cambridge, 1993).

34. I. Burghardt and P. Gaspard, J. Chem. Phys. 100, 6395 (1994).

35. K. Ohde, C. D. Pibel, A. Hishikawa, and K. Yamanouchi, to be published.

36. K. Yamanouchi and S. Tsuchiya, J. Phys. B, in press. 